\title{
1062 Echocardiographic assessment of semilunar valve incompetence useful as screening tool but unreliable in quantification: correlation with cardiac MR velocity mapping Shelby Kutty*1, Kevin K Whitehead ${ }^{2}$, Matthew A Harris², Gil Wernovsky² and Mark A Fogel ${ }^{2}$
}

Address: ${ }^{1}$ Herma Heart Center, Children's Hospital of Wisconsin, Medical College of Wisconsin, Milwaukee, WI, USA and ${ }^{2}$ Division of Cardiology, The Children's Hospital of Philadelphia, Philadelphia, PA, USA

* Corresponding author

from I Ith Annual SCMR Scientific Sessions

Los Angeles, CA, USA. I-3 February 2008

Published: 22 October 2008

Journal of Cardiovascular Magnetic Resonance 2008, I0(Suppl I):AI87 doi:I0.I 186/I532-429X-10-SI-AI87

This abstract is available from: http://jcmr-online.com/content//0/SI/AI87

(c) 2008 Kutty et al; licensee BioMed Central Ltd.

\section{Introduction}

Correlation of Echocardiography (ECHO) and Cardiac Magnetic Resonance (CMR) in the assessment of semilunar valve incompetence (SVI) have not been examined in children and young adults with congenital heart disease.

\section{Purpose}

We hypothesized that ECHO assessment has minimal predictive validity compared to quantitative CMR data and sought to compare qualitative ECHO grades with CMR regurgitant fractions $(\mathrm{RF})$.

\section{Methods}

A single center review was performed of all patients with both ECHO and CMR assessment of SVI (1/04-1/07) within 60 days of each other. The qualitative assessment (mild, moderate, severe) of SVI and RV dilation (RVd) were recorded for all patients with pulmonary incompetence (PI). SVI and LV dimension at end diastole (LVEDd) were obtained in patients with aortic incompetence (AI). $\mathrm{RF}$ was quantified by CMR using phase-contrast velocity mapping of the respective outflow tracts. ANOVA was used for statistical analysis.

\section{Results}

One hundred and twenty one patients with at least mild SVI on ECHO were identified: 78 with PI and 43 with AI. There was a statistically significant difference between the
CMR-RF of each ECHO grade of PI and AI ( $<<0.05)$. However, there was significant overlap between grades (Figure 1). Mild ECHO PI corresponded to a RF(\%)of 139 , moderate $10-50$, and severe $20-60$. For AI, mild corresponded to an RF (\%) of 0-29, moderate 1-40, and severe 5-58. Overlap was more significant at moderate and severe grades. RV dilation showed a similar relationship with PI-RF.

\section{Conclusion}

In general, ECHO grading of mild SVI corresponds to CMR-RF of less than 39\% for PI and less than 30\% for AI. However, ECHO is unreliable in precisely categorizing SVI, with a wide range of regurgitant fractions for a given ECHO grade. ECHO should be used as a screening tool to identify patients with significant SVI, and supplemented with quantitative tools such as CMR for management decisions. 


\section{Boxplots of RF by ECHO grades of SVI}

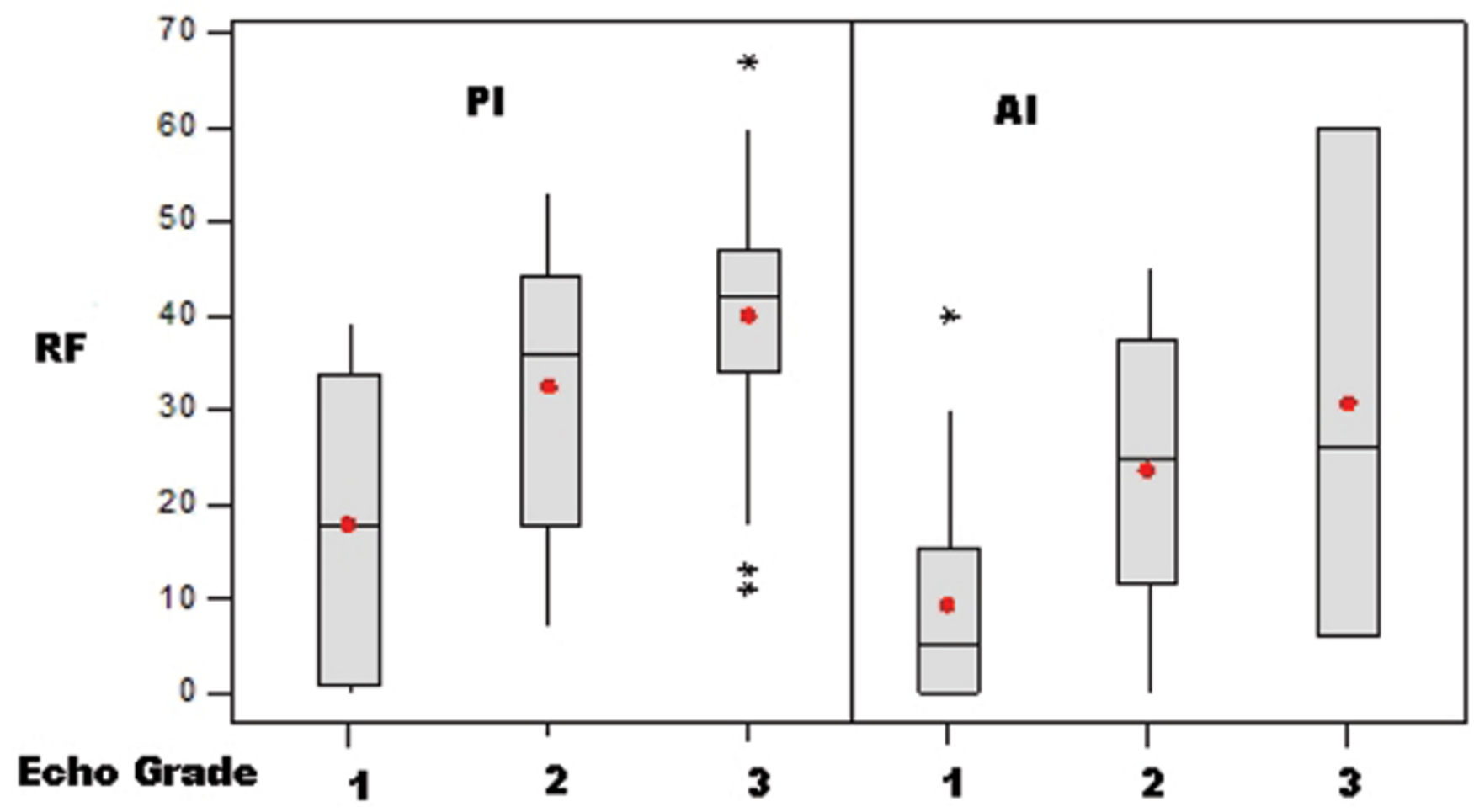

Figure I

Boxploes of RF by ECHO grades of SVI. Box plots representing the inter-quartiles ranges (first to third quartile, QI to Q3), and a line ("whiskers") extending out a distance of I.5 times the inter-quartile range below QI and above Q3. Values marked * represent the most exreme values within each group. The dot and horizontal line in each box represent the mean and median respectively. I-Mild, 2 = Moderate, $3=$ Severe. 